\title{
Early detection of poor outcome in patients with metastatic colorectal cancer: tumor kinetics evaluated by circulating tumor cells
}

This article was published in the following Dove Press journal:

OncoTargets and Therapy

13 December 2016

Number of times this article has been viewed

\author{
Virgílio Souza e Silva' \\ Ludmilla Thomé Domingos \\ Chinen ${ }^{2}$ \\ Emne A Abdallah ${ }^{2}$ \\ Aline Damascena ${ }^{2}$ \\ Jociana Paludo 3 \\ Rubens Chojniak ${ }^{3}$ \\ Aldo Lourenço Abbade \\ Dettino' \\ Celso Abdon Lopes \\ de Mello' \\ Vanessa S Alves ${ }^{2}$ \\ Marcello F Fanelli' \\ 'Department of Clinical Oncology, \\ ${ }^{2}$ International Research Center, ${ }^{3}$ Image \\ Department, A. C. Camargo Cancer \\ Center, São Paulo, Brazil
}

Correspondence: Ludmilla Thomé Domingos Chinen

International Research Center,

A. C. Camargo Cancer Center,

Rua Taguá, 440, São Paulo 01508-010,

Brazil

Tel +55 II 21892993

Email ludmilla.chinen@cipe.accamargo. org.br
Background: Colorectal cancer (CRC) is the third most prevalent cancer worldwide. New prognostic markers are needed to identify patients with poorer prognosis, and circulating tumor cells (CTCs) seem to be promising to accomplish this.

Patients and methods: A prospective study was conducted by blood collection from patients with metastatic CRC (mCRC), three times, every 2 months in conjunction with image examinations for evaluation of therapeutic response. CTC isolation and counting were performed by Isolation by Size of Epithelial Tumor Cells (ISET).

Results: A total of 54 patients with mCRC with a mean age of 57.3 years (31-82 years) were included. Among all patients, $60 \%(\mathrm{n}=32)$ were carriers of wild-type KRAS (WT KRAS) tumors and $90 \%$ of them $(n=29)$ were exposed to monoclonal antibodies along with systemic treatment. Evaluating CTC kinetics, when we compared the baseline (pretreatment) CTC level (CTC1) with the level at first follow-up (CTC2), we observed that CTC1-positive patients (CTCs above the median), who became negative (CTCs below the median) had a favorable evolution ( $\mathrm{n}=14)$, with a median progression-free survival (PFS) of 14.7 months. This was higher than that for patients with an unfavorable evolution (CTC1 - that became CTC2+; $\mathrm{n}=13,6.9$ months; $P=0.06$ ). Patients with WT KRAS with favorable kinetics had higher PFS (14.7 months) in comparison to those with WT KRAS with unfavorable kinetics (9.4 months; $P=0.02)$. Moreover, patients whose imaging studies showed radiological progression had an increased quantification of CTCs at CTC2 compared to those without progression $(P=0.04)$.

Conclusion: This study made possible the presentation of ISET as a feasible tool for evaluating CTC kinetics in patients with $\mathrm{mCRC}$, which can be promising in their clinical evaluation.

Keywords: circulating tumor cells, metastatic colorectal cancer, kinetics, ISET

\section{Introduction}

Colorectal cancer (CRC) is the third most incident cancer around the world. With an estimated 1.36 million new cases per year and $\sim 600,000$ deaths related to the disease, $\mathrm{CRC}$ is a challenging malignancy. ${ }^{1}$ Around $50 \%-60 \%$ of patients diagnosed with CRC will eventually develop metastasis and $30 \%$ will present with metastatic disease at the moment of diagnosis. ${ }^{2-4}$ The last decade was characterized by progress in the availability of new drugs with distinct mechanism of action and a positive impact on sequential treatment of metastatic CRC (mCRC), including important gains in median survival rates for these patients, which currently exceed 24 months. ${ }^{5-9}$ The availability of new drugs has the potential to bring improvement in the survival rates. However, monoclonal antibodies, such as cetuximab/panitumumab (anti-EGFR antibodies) and bevacizumab (anti-VEGF antibody), depend on molecular characteristics of each tumor. ${ }^{10}$ As a rule, anti-EGFR 
antibodies are used only in patients with wild-type RAS. RAS is an important protein in EGFR signaling pathway; mutations can constitutively activate the protein, conferring a higher proliferation rate for tumor cells and resistance to anti-EGFR blockage. ${ }^{11}$ Despite gains in survival rates, the increased cost of the treatment is not directly reflected in the improvement of health and well-being of patients or in the ability to identify ideal treatments for specific patients.

Many prognostic factors have been associated with outcome in CRC, such as performance status, age, gender, weight loss, an increase in carcinoembryonic marker levels, and number of metastatic sites, among others. ${ }^{12,13}$ Several studies have attempted to identify biomarkers to improve the standard prognostic factors. ${ }^{14}$ The goal is to individualize therapeutic strategies and to identify patients with worse prognosis or those patients with rapid progression of disease, and who could ultimately benefit from a more aggressive therapy. ${ }^{15-17}$

Circulating tumor cell (CTC) studies have demonstrated promising results in the segment of patients with metastatic cancer. ${ }^{18}$ Various recent studies have shown CTC levels as predictors of progression-free survival (PFS) and overall survival (OS) in patients with early and metastatic breast cancer, and also metastatic prostate cancer. ${ }^{19-24}$ In patients with $\mathrm{mCRC}$, some publications describe a correlation of the CTC levels and prognosis. ${ }^{25-27}$ The presence of at least 3.0 CTCs/7.5 mL of blood at the diagnosis and at follow-up provides an independent prognostic factor for worse PFS and OS. ${ }^{28}$ These values, when used in conjunction with imaging examinations, could provide additional diagnostic information. ${ }^{29}$ There are, basically, three methods to separate CTCs, as follows: 1) immunological (based on capture by antibodies), such as CellSearch ${ }^{\circledR}$ System (Veridex, LLC, NJ, USA); 2) based on physical properties of cells (eg, ISET ${ }^{\circledR}$, Rarecells, Paris, France), and 3) the functional assays, such as EPISPOT. The isolation by size of epithelial tumors (ISET) technology is a physical-based assay that consists of separating cells by size. It has an advantage of isolating CTCs in a marker-independent manner. Thus, if these cells have downregulated and have no expression of epithelial markers (EpCAM and cytokeratin [CK]), they will not be lost by Isolation by Size of Epithelial Tumor Cells (ISET). ${ }^{29}$ In comparison with CellSearch ${ }^{\circledR}$ System, it seems that ISET can capture a larger amount of tumor cells than CellSearch, without risk of overestimate counts, as this last one system which can also capture other non-malignant cells expressing EpCAM and CK. To date, the majority of studies with CTC kinetics were carried out using the CellSearch ${ }^{\circledR}$ System. ${ }^{30-33}$
Therefore, the purpose of our study was to evaluate the clinical evolution and therapeutic response, using conventional image examinations and CTC counts, done by a size-based isolation method (ISET) in patients with $\mathrm{mCRC}$ in sequential lines of treatment.

\section{Patients and methods}

\section{Patients}

Enrollment in the study was limited to patients who signed an informed consent form. This study was approved by the local research ethics committee (protocol number: 1367-10). All participants had $8 \mathrm{~mL}$ of blood collected at every scheduled visit. Patients were included according to the following criteria: 18 years of age or older; ECOG 0-2 without organ dysfunction; metastatic disease confirmed by pathological and/or radiological analysis; chemotherapy regimen for metastatic disease; the extent of the disease determined by a physical examination and by imaging and disease measureable by Response Evaluation Criteria in Solid Tumors (RECIST) criteria Version 1.1. ${ }^{34}$

\section{Assay methods}

We used ISET for analysis and characterization of CTCs. The peripheral blood of patients with $\mathrm{mCRC}$ was collected in ethylenediaminetetraacetic acid tubes $(8.0 \mathrm{~mL})$ and maintained at room temperature, under homogenization, for not $>4$ hours. The samples were diluted (1:10) in ISET Buffer ${ }^{\circledR}$. After 10 minutes of incubation, the samples were filtered, washed with phosphate buffered saline, and the membranes were removed from the ISET Block ${ }^{\circledR}$ and, after drying in the dark at room temperature, stored at $-20^{\circ} \mathrm{C}$, until analysis.

To count CTCs, four ISET membrane spots were cut and submitted to immunocytochemistry with anti-CD45 antibody (1:100; clone 2B11+PD7/26, Dako ${ }^{\mathrm{TM}}$, Carpinteria, California, USA), a surface leukocyte common antigen, in order to rule out miscounting of CTCs. The assay was performed as described previously by Chinen et al. ${ }^{35}$ After assembly, slides were examined under a white light microscope. The CTCs were characterized based on the following criteria: negative cells for CD45, nucleus size $\geq 12 \mu \mathrm{m}$, hyperchromatic and irregular nucleus, visible presence of cytoplasm, and a high nucleus-cytoplasm ratio $(>50 \%) .{ }^{30}$

\section{Study design}

This was a prospective study conducted by whole blood collection from patients with $\mathrm{mCRC}$ treated at A. C. Camargo Cancer Center, São Paulo, Brazil. The patients' blood was collected at three different times: baseline (after the detection 
of metastasis or tumor progression, when a new chemotherapy regimen was initiated) and two additional follow-up visits (every 2 months) in an attempt to reconcile CTC collections with routine imaging for therapeutic response evaluation.

Patients were evaluated using computed tomography and/or magnetic resonance imaging of the thorax, abdomen, and pelvis before the first collection of CTCs, as well as approximately every 2-4 months during treatment, or as indicated by the treating physician. The images were interpreted using RECIST criteria Version $1.1^{34}$ and classified as complete response, partial response, stable disease, or progression of disease.

\section{Statistical analysis methods}

The distributions of absolute and relative frequencies were made for the clinical-pathological variables and also the qualitative treatment and the mean and SD for age. The Kaplan-Meier estimator and the log-rank test were used in the analysis of PFS. Variance analysis of repeated measurements was used to access CTC evolution during the image evaluations. The level of significance adopted was $<5 \%$ and the analyses were performed in the program SPSS for Windows Version 15.

\section{Results \\ Patients}

Between July 2012 and December 2013, we recruited 54 patients diagnosed with mCRC. The mean age was 57.3 years ( $31-82$ years), and most of the patients were male $(n=31 ; 59 \%)$. At the time of the inclusion, all patients had metastatic disease, including $65 \%(n=35)$ who had at least one metastatic site and $7 \%(n=4)$ with more than two metastatic sites. Only $30 \%(n=16)$ of patients were treated with first-line chemotherapy at the time of enrollment in the study. Most of them had received previous treatment prior to the study. Patients with wild-type KRAS (WT KRAS) constituted $60 \%(n=32)$ of the study population and during follow-up, $90 \%(n=29)$ of them were exposed to monoclonal antibodies combined with systemic treatment (Table 1).

\section{CTC counts}

The quantitative analysis of CTCs by the ISET was made at three distinct times during follow-ups for each patient: baseline (CTC1), first follow-up performed between the 6th and the 8th week of treatment (CTC2), and the second follow-up done between the 12th and 16th week of treatment (CTC3). Because there is not yet a cutoff value for counting CTCs detected by ISET, we set a standard using the median number of CTCs
Table I Characteristics of the studied population

\begin{tabular}{ll}
\hline Characteristics & $\mathbf{N}(\%)$ \\
\hline Average age (minimum-maximum), years & $57.3(3 \mathrm{I}-8 \mathrm{I})$ \\
Gender & \\
Male & $3 \mathrm{I}(59)$ \\
Female & $23(4 \mathrm{I})$ \\
KRAS (data available in 53/54 patients) & \\
$\quad$ Wild-type & $32(60)$ \\
Mutant & $21(40)$ \\
Use of antibodies & \\
No & $25(46)$ \\
Bevacizumab & $19(36)$ \\
Cetuximab & $10(18)$ \\
Liver metastasis & \\
Yes & $25(46)$ \\
No & $29(54)$ \\
Number of metastatic sites & \\
I & $35(65)$ \\
2 & $15(28)$ \\
$>2$ & $4(7)$ \\
Lines of treatment, pre-CTCI & \\
0 & $16(30)$ \\
I & $20(36)$ \\
$\geq 2$ & $18(34)$ \\
\hline
\end{tabular}

Abbreviation: CTC, circulating tumor cell.

from all patients evaluated each time. As a consequence, the cutoff for CTC1 was $2.0 \mathrm{CTCs} / \mathrm{mL}(0-31.0 \mathrm{CTCs} / \mathrm{mL})$, for CTC2 was 3.0 CTCs $/ \mathrm{mL}(0-47.0 \mathrm{CTCs} / \mathrm{mL})$, and for CTC3 was 3.0 CTCs $/ \mathrm{mL}(0-77.0 \mathrm{CTCs} / \mathrm{mL})$. Patients with CTC values below the established cutoffs were considered as patients with negativity for CTC (CTC-), and equal to or higher, as patients with positivity for CTC (CTC+). Illustrative pictures of CTCs and leukocytes are shown in Figure 1. Out of the 54 patients enrolled in the study, 33 (62\%) had the three blood samples collected for analysis (CTC1, CTC2, and CTC3). Baseline sample (CTC1) was not obtained for one patient (the sample was collected but the blood coagulated).

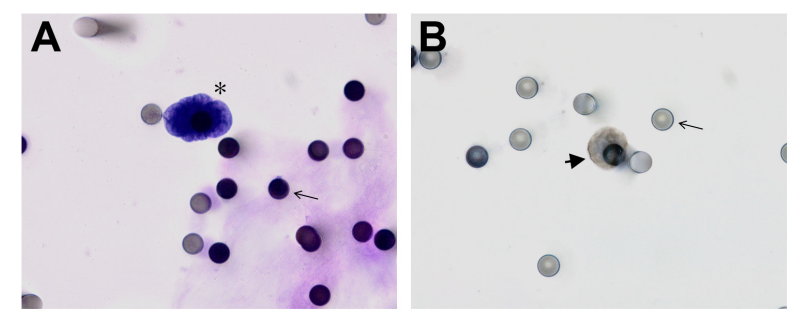

Figure I Cells isolated by ISET.

Notes: (A) CTC visualized by hematoxylin-eosin. (B) Immunostaining of leukocyte with CD45 and counterstaining with DAB. Asterisk indicates a CTC; thin arrows represent pores of ISET membrane; and the thick arrow shows a leukocyte. Both images were taken at $\times 600$ magnification using a light microscope (Research System Microscope BX6I - Olympus, Tokyo, Japan) coupled to a digital camera (SCI00 - Olympus, Tokyo, Japan).

Abbreviations: CTC, circulating tumor cell; DAB, 3,3'-daminobenzidine; ISET, isolation by size of epithelial tumors. 
First follow-up (CTC2) sample was not obtained from 12 patients and second follow-up (CTC3) sample was not collected from 21 patients. The reasons for not obtaining the samples (CTC2 and CTC3) were mostly due to the patient not consenting to a new blood draw, loss of follow-up, or death. There was no correlation between CTC values and any clinical-demographic characteristics.

\section{CTC analysis and its prognostic value}

The CTC values monitored at three serial collections allowed the analysis of CTC kinetics. Comparing the patients' baseline CTC level (CTC1) with the level at first follow-up (CTC2), patients with positivity for CTC at baseline who became negative were classified with a favorable evolution (CTC1+ and CTC2-; n=14) and had a PFS median of 14.7 months. This was higher than PFS of patients with an unfavorable evolution (CTC1- and CTC2+; n=13), who had negativity for CTC at baseline and became positive in CTC2 with a median PFS of 6.9 months ( $P=0.06$; Figure 2$)$. In patients without modification in the kinetics of the CTCs

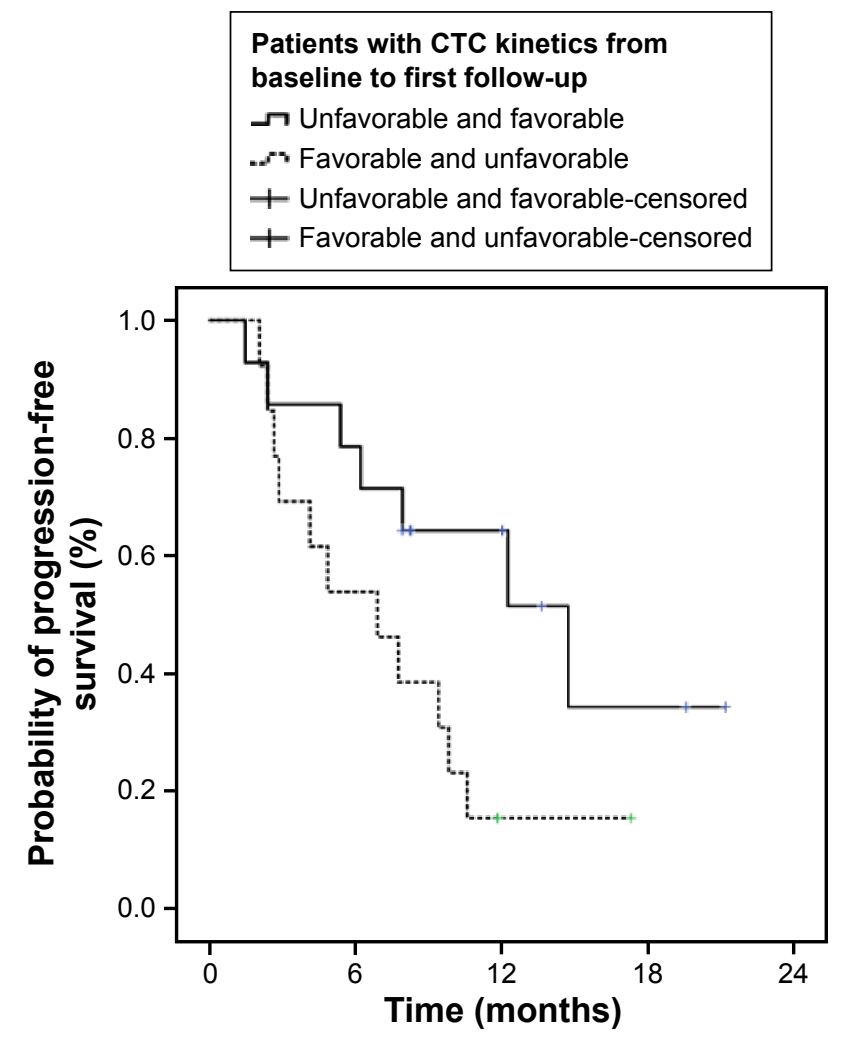

Figure 2 Progression-free survival evaluation in patients with CTC kinetic alteration on the first two analyses (CTCI and CTC2).

Notes: Continuous line: positive patients with favorable kinetics and prognosis $(\mathrm{CTCl}+$ and $\mathrm{CTC2}-)$. Dotted line: patients with unfavorable kinetics and prognosis ( $\mathrm{CTCl}-$ and $\mathrm{CTC2+;} P=0.06$ ).

Abbreviation: CTC, circulating tumor cell.
(CTC1+ and $\mathrm{CTC} 2+$ or CTC1- and CTC2-), there was no difference between the PFS curves (10.3 and 10.4 months, respectively; $P=0.6$; Figure 3 ). However, for those patients with no change in CTC kinetics (based on established cutoff), we observed that the pure analysis of CTC counts could help to define prognosis in mCRC. Patients who had increased CTC numbers during follow-up showed the worst prognosis in relation to those who had a decrease in CTC counts (Table 2). The correlation between the second collection and the third collection did not demonstrate a statistically significant difference $(P=0.79)$. Figure 4 shows the radiological evolution (disease progression) with an increase in CTC counts in a patient (patient 1) of the study, to demonstrate the data presented. We also made the stratification of groups based on quantification of CTCs at three times (CTC1, CTC2, and CTC3) in relation to disease progression, as shown in Figure 5. The dot plot figure shows CTCs from each patient at each time of blood collection.

Based on the CTC kinetic profile, we correlated the favorable/unfavorable patients with the mutational status of $K R A S$. Patients with mutant KRAS (MT KRAS) and favorable

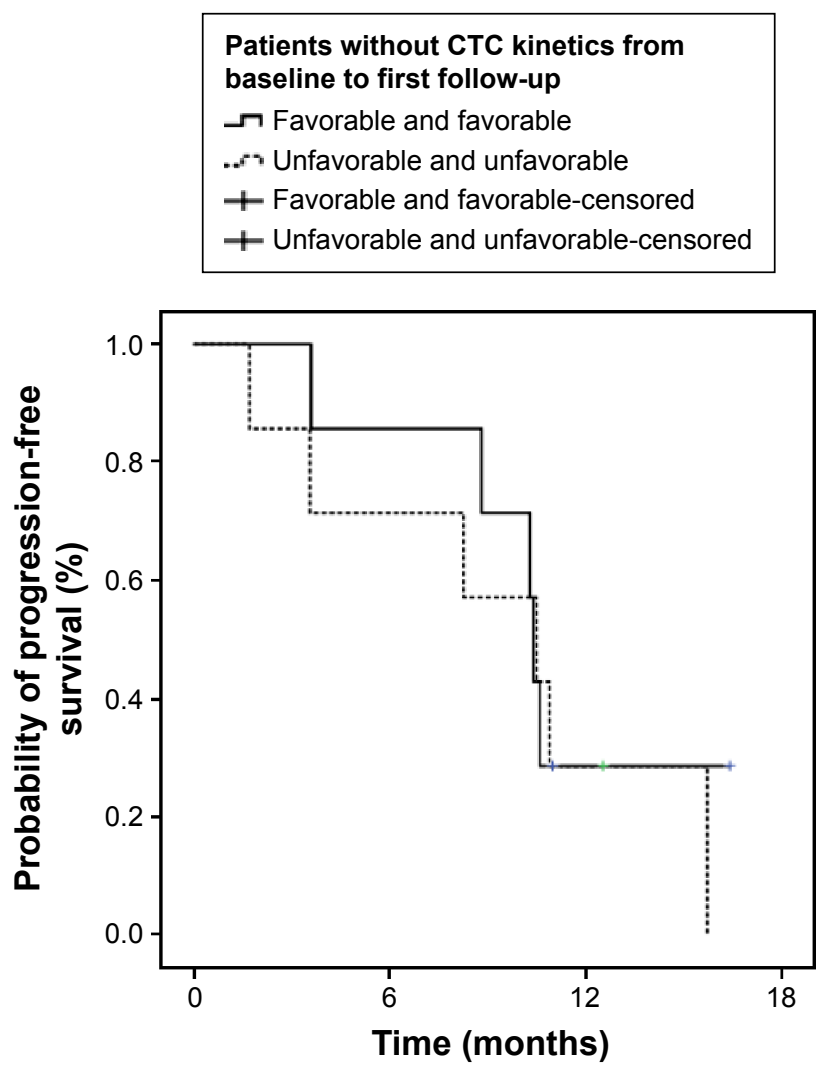

Figure 3 Progression-free survival evaluation in months, in patients without kinetic alteration of $\mathrm{CTCs}(\mathrm{CTCl}+$ and $\mathrm{CTC} 2+)$ and $(\mathrm{CTCl}-$ and $\mathrm{CTC2}-)$.

Notes: Continuous line: patients with positive CTC maintenance. Dotted line: patients with negative $C T C$ maintenance $(P=0.60)$.

Abbreviation: CTC, circulating tumor cell. 
Table 2 Individual values of CTC counts of patients whose CTC kinetics based on established cutoff did not show alteration

\begin{tabular}{|c|c|c|c|c|c|c|}
\hline Patient ID & $\begin{array}{l}\text { CTCI } \\
\text { (CTCs/mL) }\end{array}$ & $\begin{array}{l}\text { CTCI } \\
\text { (+ or }- \text { ) }\end{array}$ & $\begin{array}{l}\text { CTC2 } \\
\text { (CTCs/mL) }\end{array}$ & $\begin{array}{l}\text { CTC2 } \\
(+ \text { or }-)\end{array}$ & $\begin{array}{l}\text { Kinetics evolution } \\
\text { (CTCI and CTC2) }\end{array}$ & $\begin{array}{l}\text { PFS } \\
\text { (months) }\end{array}$ \\
\hline Patient $\mathrm{A}$ & 46.0 & + & 6.0 & + & NA & 11.4 \\
\hline Patient B & 11.0 & + & 31.0 & + & NA & 5.4 \\
\hline Patient C & 4.0 & + & 24.0 & + & NA & 2.7 \\
\hline Patient D & 0 & - & 0 & - & NA & NP \\
\hline Patient E & 2.0 & - & 1.0 & - & NA & $\mathrm{NP}$ \\
\hline Patient F & 12.0 & + & 11.0 & + & NA & NP \\
\hline Patient G & 29.0 & + & 3.0 & + & NA & 4.3 \\
\hline Patient $\mathrm{H}$ & 0 & - & 0 & - & NA & 6.9 \\
\hline Patient I & 0 & - & 0 & - & NA & 10.4 \\
\hline Patient J & 4.0 & + & 11.0 & + & NA & 6.6 \\
\hline Patient K & 8.0 & + & 3.0 & + & NA & 3.5 \\
\hline Patient L & 1.0 & - & 1.0 & - & NA & 4.4 \\
\hline Patient M & 0 & - & 1.0 & - & NA & 10.5 \\
\hline Patient N & 3.0 & + & 8.0 & + & NA & II.I \\
\hline
\end{tabular}

Abbreviations: CTC, circulating tumor cell; PFS, progression-free survival; NA, no alteration; NP, no progression.

CTC kinetic evolution ( $\mathrm{n}=3$ ) had a PFS of 5.3 months, higher than that of the patients with MT KRAS with unfavorable CTC kinetic evolution ( $\mathrm{n}=4 ; 2.4$ months), although without reaching statistical significance $(P=0.6)$. In addition, the patients with WT KRAS with a favorable trend $(\mathrm{n}=11)$ showed a median PFS of 14.7 months, higher than that of the patients with WT KRAS with unfavorable kinetics $(\mathrm{n}=9)$, of 9.4 months $(P=0.02$; Figure 6$)$.

In relation to the treatment with anti-angiogenic therapies and CTC kinetic profile, patients who used anti-VEGF treatment who presented favorable CTC kinetics $(n=6)$ had a PFS of 18.2 months, higher than those with unfavorable kinetics ( $\mathrm{n}=5$; PFS $=5.6$ months; $P=0.007$; Figure 7 ). In addition to the quantification of CTCs, patients were also analyzed through the evaluation of images for each CTC collection up to 4 months after the CTC 3 collection. We observed that the patients whose image examinations showed radiological progression consistent with RECIST criteria ${ }^{34}$ (20/32) demonstrated higher average CTC quantification at the second time (CTC2) compared with those that did not show progression in the imaging examinations (12/32), and this difference was statistically significant ( $P=0.04$; Figure 8). To illustrate this correlation, the radiological evolution and CTC count from patient 2 are shown in Figure 4, where CTC elevation at the second time correlated with evidence of disease progression on image examinations done after the CTC3 collection.

\section{Discussion}

Treatment of patients with $\mathrm{mCRC}$ in the past 10 years has become substantially more complex. The prolonged survival of patients has become possible due to the improvement in the therapeutic arsenal composed of the following: fluoropyrimidine, oxaliplatin, irinotecan and targeted therapies (bevacizumab, panitumumab, cetuximab, regorafenib, and aflibercept) and, in some situations, surgery for metastasis. ${ }^{29,36}$ From this point, oncologists need useful tools to evaluate the intensity and the best sequence of systemic treatment in order to provide patients the access to all potentially effective drugs during the evolution of the patient's disease. Defining the initial treatment with one, two, or three medications associated or not with biological agents (monoclonal antibodies and tyrosine kinase inhibitors) becomes more difficult due to clinical and pathological complexities of $\mathrm{mCRC}$ and the lack of non-radiological markers that are useful for defining therapeutic strategies. In this way, evaluating the dynamics of CTCs could guide the oncologists in choosing a systemic therapy and predicting the clinical response from favorable and unfavorable patients. ${ }^{37,38}$

Our study showed that the CTC kinetics could be used as a prognostic parameter in $\mathrm{mCRC}$. We could differentiate patients with a favorable outcome with a median PFS of 14.7 months from unfavorable patients with a median PFS of 6.9 months $(P=0.06)$. We attributed the non-statistically significant difference to the small sample size and to the heterogeneity of the studied population. However, besides this, our results were similar to a recently published study that demonstrates a correlation of CTC kinetics in metastatic breast cancer $(\mathrm{mBC})$ with prognosis and evaluation of response to the treatment adopted. ${ }^{39}$ This study with $356 \mathrm{mBC}$ patients demonstrated that those with favorable CTC kinetics had a higher propensity to respond to 


\section{Patient 1}

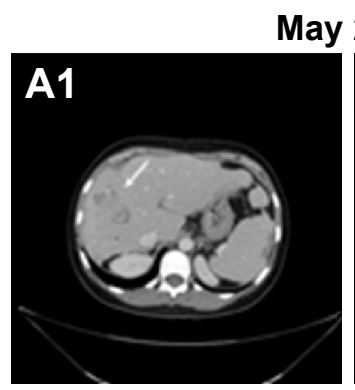

\begin{tabular}{|c|c|}
\hline Month, year & CTC count \\
\hline June, 2013 & $1.0 \mathrm{CTC} / \mathrm{mL}$ \\
\hline
\end{tabular}

Patient 2

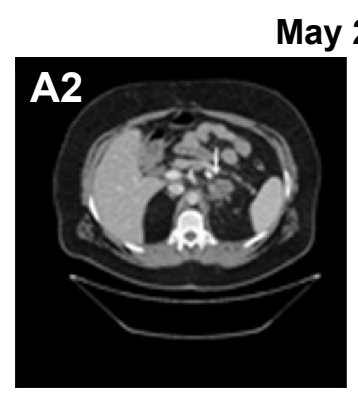

\begin{tabular}{|l|l|}
\hline Month, year & CTC count \\
\hline June, 2013 & $1.0 \mathrm{CTC} / \mathrm{mL}$ \\
\hline July, 2013 & $36.0 \mathrm{CTC} / \mathrm{mL}$ \\
\hline September, 2013 & $2.0 \mathrm{CTC} / \mathrm{mL}$ \\
\hline
\end{tabular}

\section{September 2013}

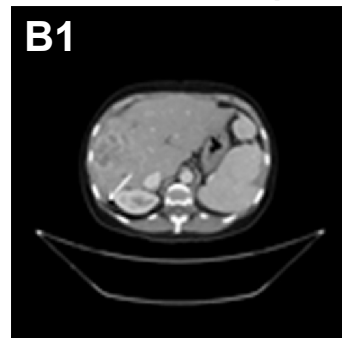

\begin{tabular}{|l|l|}
\hline Month, year & CTC count \\
\hline August, 2013 & $46.0 \mathrm{CTC} / \mathrm{mL}$ \\
\hline October, 2013 & $76.0 \mathrm{CTC} / \mathrm{mL}$ \\
\hline
\end{tabular}

\section{September 2013}

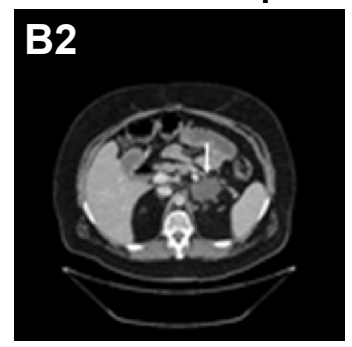

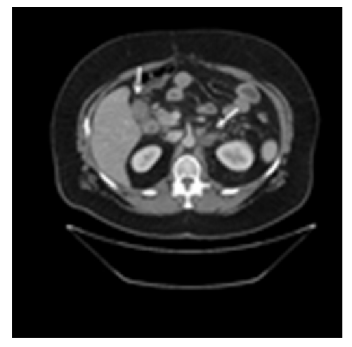

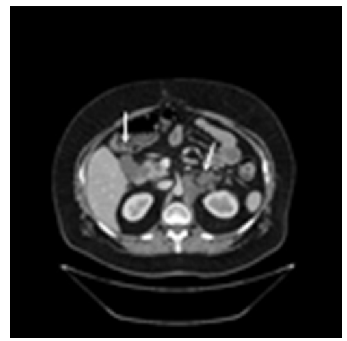

November 2013
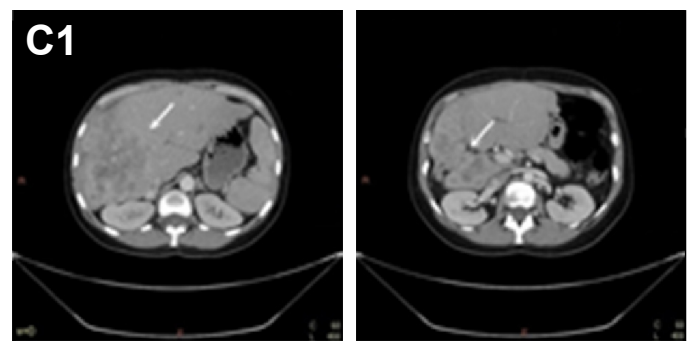

Figure 4 Radiological evolution and CTC counting.

Notes: Patient I: (AI): image of hepatic lesion evidence (arrows) obtained before the collection of CTCI (May 20I3); (BI): image (September 20I3) of hepatic lesion evidence (arrows) in progression when image Al compared with image after the collection of CTC2; (CI): image of hepatic lesion evidence (arrows) in progression when compared with image B I obtained after collection of CTC3 (November 2013). Patient 2: (A2): image of lymph node involvement evidence (arrows) obtained before the collection of CTCI (May 20I3); (B2): image (September 2013) of evidence of progression of lymph node disease (arrows) compared with illustration A2.

Abbreviation: CTC, circulating tumor cell.

the proposed treatment, supporting the evaluation that the dynamic counting of CTCs could better reflect the prognosis and the aggressiveness of the disease than the isolated counting of cells. ${ }^{37}$ Cohen et a ${ }^{28,40}$ in their publications on mCRC using CellSearch ${ }^{\circledR}$ System also demonstrated the importance of CTC kinetics with evidence of increased survival for patients with conversion from unfavorable to favorable CTC condition. In addition, to support our results, a meta-analysis published in $2014^{41}$ suggested that patients with mCRC who converted from CTC-low to CTC-high or who were persistently CTC-high during treatment had an unfavorable disease (disease progression) compared with 


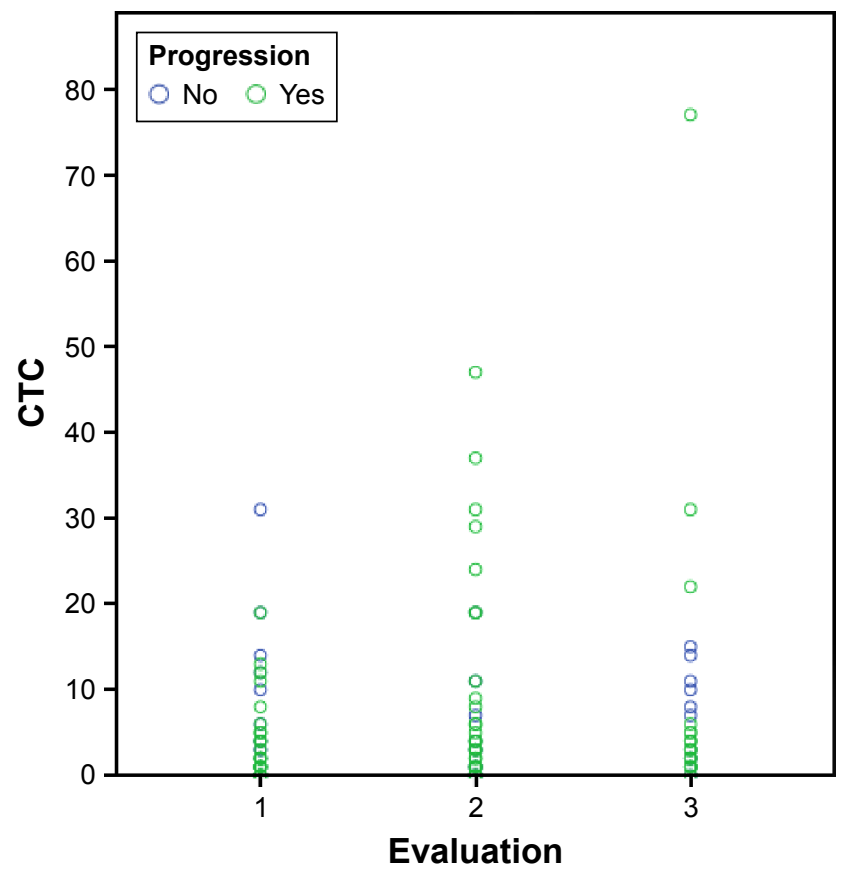

Figure 5 Dot plot figure.

Notes: Each circle represents a patient analyzed three times: baseline (CTCI), first follow-up (CTC2), and second follow-up (CTC3). Blue circles refer to the absence of disease progression and the green ones to the presence.

Abbreviation: CTC, circulating tumor cell.

those patients who converted from CTC-high to CTC-low (odds ratio $=27.088,95 \%$ CI [4.960-147.919], $P<0.001$ ). Therefore, we might consider the possibility that the evaluation of CTC kinetics for patients with $\mathrm{mCRC}$ could become an important tool and could be pursued in further studies in order to improve clinical practice. However, different from our study, all the included studies in the meta-analysis used methods with low sensitivity and specificity to isolate CTCs (immunological or reverse transcription polymerase chain reaction techniques).

We were the first to use ISET, a highly sensitive and specific method, to evaluate CTC kinetics in patients with mCRC. Although still not approved by the US Food and Drug Administration, like the CellSearch ${ }^{\circledR}$ System, it has passed through technical ${ }^{30,42}$ and clinical ${ }^{43}$ validations and has the advantage of allowing cell visualization and quantification. ISET is an independent method to isolate CTCs, which allows their cytopathological identification. It is based on the principle that tumor cells from solid tumors that are much larger than leukocytes, with nuclei of $24 \mu \mathrm{m}$ or larger, can be retained on the ISET filter with a pore size of $8 \mu \mathrm{m}$, larger than the majority of leukocytes. ${ }^{44}$ As ISET is a size-based method, it does not depend on antigen markers and is not expected to have selection bias and false-positive (epithelial non-tumor cells detected as tumor cells in healthy patients)

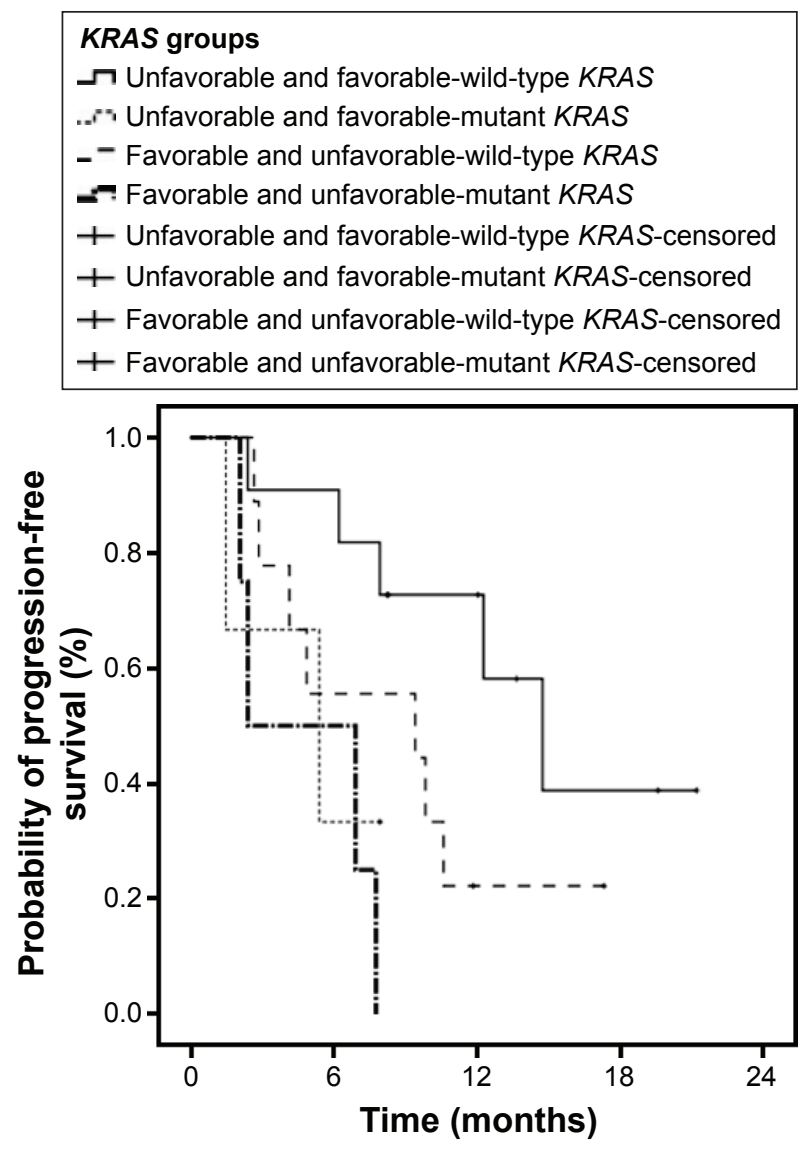

Figure 6 Progression-free survival based on CTC kinetics and the status of the KRAS mutation.

Notes: Continuous line: patients with wild type KRAS and favorable kinetics ( $\mathrm{CTCl}+$ and $\mathrm{CTC2}-;$ 14.7 months). Dotted line: patients with mutant KRAS and favorable kinetics ( $\mathrm{CTCl}+$ and $\mathrm{CTC2}-; 5.3$ months). Dashed line: patients with wildtype and unfavorable kinetics (CTCl- and $\mathrm{CTC2+;} 9.4$ months). Dashed-dotted line: patients with mutant KRAS and unfavorable kinetics (CTCI- and $\mathrm{CTC2+;}$ 2.4 months) $(P=0.02)$

Abbreviation: CTC, circulating tumor cell.

and false-negative (CTCs with mesenchymal markers not detected) results, as observed with CellSearch. ${ }^{44}$

We could classify patients as with favorable and unfavorable evolution based on median CTC number of each phase, even though there is no standard cutoff value for ISET; our data were comparable with the results of studies that used standardized techniques such as CellSearch ${ }^{\circledR}$ System (Veridex). ${ }^{16,24,26,27,45,46}$ However, we believe that the most important in terms of clinical practice is to observe each patient individually, independently of values of cutoff, and for this purpose the ISET method would be more appropriate, as it would enable the quantification of CTCs with better accuracy. As CTCs can be counted per milliliter of blood by ISET, this allows a better follow-up of patients, based on increase or decrease of their own absolute CTC counts.

Upon evaluating the KRAS status of patients included in our study, we found that those MT KRAS with unfavorable 


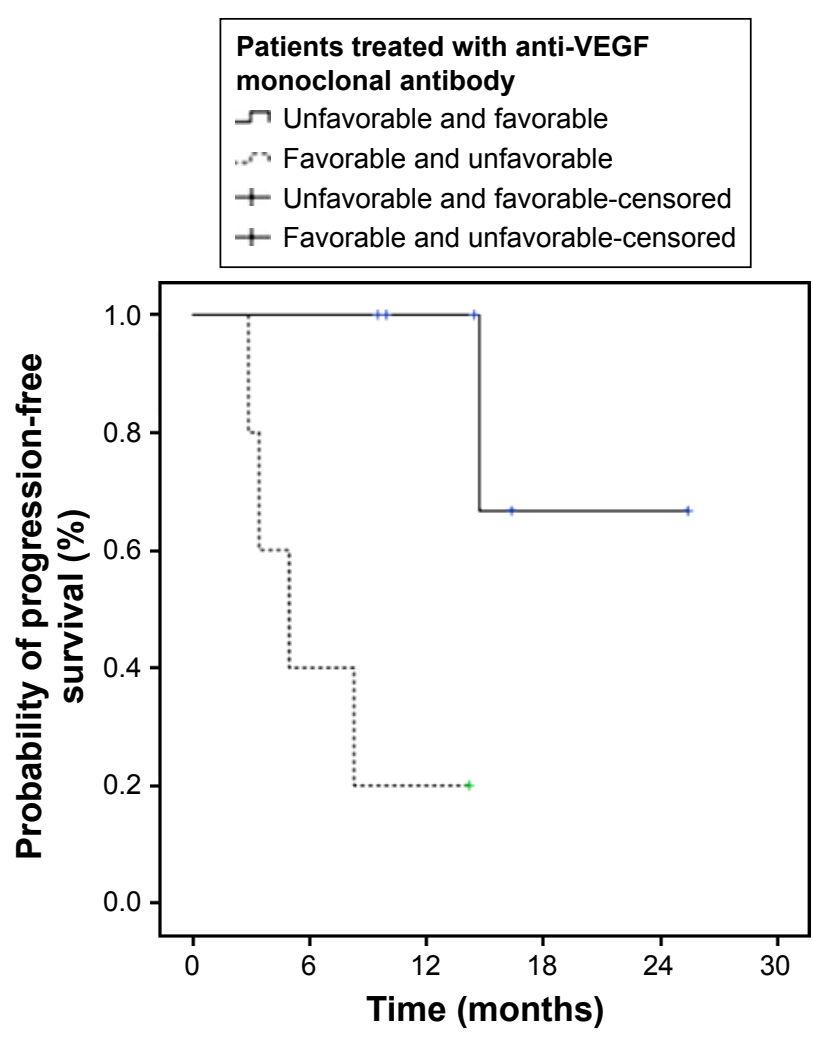

Figure 7 Progression-free survival based on CTC kinetics and the treatment with anti-VEGF monoclonal antibody.

Notes: Continuous line: patients treated with anti-VEGF with favorable kinetics (CTCI+ and CTC2-). Dotted line: patients treated with anti-VEGF with unfavorable kinetics ( $\mathrm{CTCl}-$ and $\mathrm{CTC2+;} P=0.007)$.

Abbreviation: CTC, circulating tumor cell.

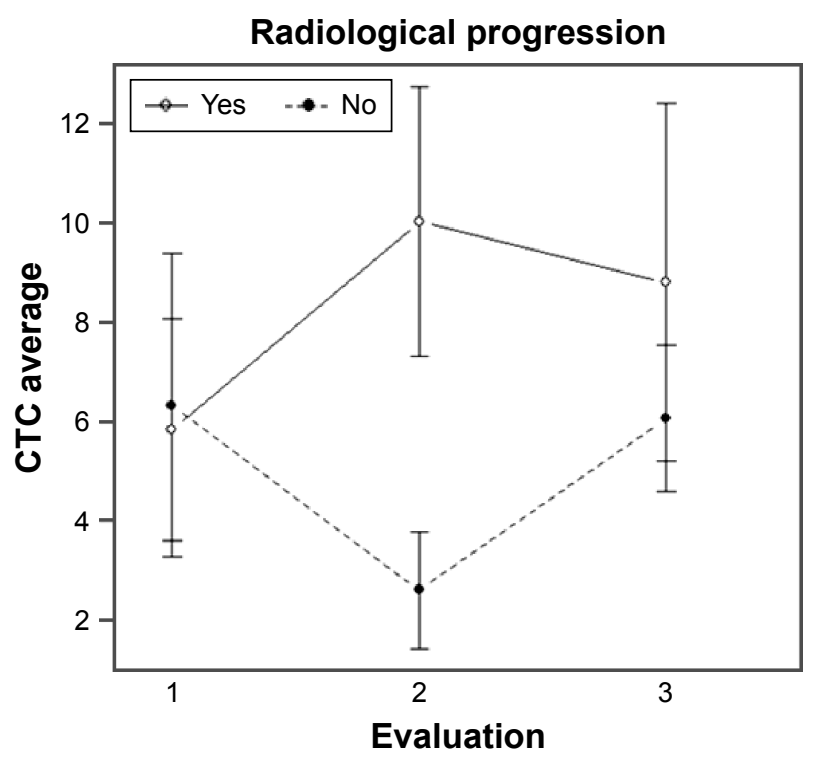

Figure 8 Correlation between CTC quantification and radiological progression. Notes: Continuous line: patients with radiological progression and increased CTC quantification in the second collection (CTC2). Dotted line: patients without radiographic progression who did not have higher CTC in the second collection (CTC2; $P=0.04$ ).

Abbreviation: CTC, circulating tumor cell. kinetics had a worse median PFS (2.4 months) compared with those with MT KRAS and favorable kinetics (median PFS of 5.3 months, $P=0.02$ ). Although the sample for this analysis was restricted to 27 patients, it is important to correlate these data with the study ${ }^{45}$ that evaluated the first-line treatment of 1589 patients with mCRC. In this study, the first count higher than $3.0 \mathrm{CTCs} / 7.5 \mathrm{~mL}$, in association with the KRAS mutation, presented worse prognosis when these patients were compared with patients with CTC $<3.0 / 7.5 \mathrm{~mL}{ }^{47}$ This correlation allows us to support the importance of the CTC kinetics not addressed in this study.

Another important point that should be investigated in further studies is the evaluation of the WT KRAS subgroup with unfavorable kinetics who presented a median PFS of 9.4 months, less than the subgroup of patients with WT KRAS and favorable kinetics who had a PFS of 14.7 months $(P=0.02)$. These results demonstrate the ability to sub-classify groups who would benefit from treatment with EGFR blockade, by CTC kinetics. So, we are able to question if this therapeutic choice in the context of its toxicities and costs could provide substantial benefits for such patients. These data allow us to say that within the $K R A S$ subgroups (mutant and non-mutant) it is possible to distinguish the evolution of patients based on CTC kinetics and, with this, define therapeutic strategies designed for patients with worse prognosis. It is important to point out that since 2013 the extended RAS testing is of clinical standard; ${ }^{48}$ however, at the time of enrollment of this study we did not have this knowledge, justifying the study focus only on $K R A S$.

Finally, another important issue that should be investigated in further studies is the evaluation of treatment with anti-angiogenic therapies that was possible through the use of ISET technique. Although with a limited number of patients, we are the first group to demonstrate that by ISET it is possible to follow up patients under treatment with anti-angiogenic therapies. Other authors have demonstrated that the CellSearch ${ }^{\circledR}$ System suffers a mask in CTC counts when patients receive those treatments. ${ }^{49-51}$ Probably, it happens because CTCs from patients who are treated with anti-angiogenic therapies lose their epithelial markers, the targets of CellSearch. A recently published study with a CRC cell lineage ${ }^{52}$ showed that the chronic exposure of these cells to bevacizumab (BEV) induced decreased expression of EpCAM $40 \mathrm{kDa}$ isoform and increased expression of EpCAM $42 \mathrm{kDa}$ isoform, together with a decreased expression of CKs, markers commonly used by CellSearch. The recovery rate of cells through this system was gradually reduced in the course 
of treatment with BEV, being $84 \%, 70 \%$, and $40 \%$ at 1,2 , and 3 months, respectively.

Finally, our study demonstrated that CTC counts could help to infer disease progression before imaging examinations. Here, patients who had disease progression as seen from imaging during follow-ups, presented, on average, higher CTC counts in the first follow-up $(P=0.04)$. This makes it possible to carefully monitor patients and discuss the modification of early therapeutic plans. In this way, the analysis of CTC kinetics can help in the choice of treatment ${ }^{25,29,53}$ and help to modify supposedly ineffective therapeutic schemes, avoiding possible unnecessary side effects and to institute new therapeutic regimes early, in order to better control the patient's disease. Still, for patients with unfavorable kinetics or those who present higher CTC levels during evolution, more intensive clinical follow-up would be crucial. We hope that in a few years, more standardized protocols with higher sensitivity and specificity to isolate and to analyze CTCs will help the clinician to identify those patients with progression of disease and anticipate a better treatment based on the elevation of biological markers provided by CTC tests.

\section{Conclusion}

Despite the limited sample size and the limited follow-up, our study could demonstrate the feasibility of counting CTCs from patients with mCRC with ISET (Rarecells), which in its simplicity bears huge advantages for clinical implementation. In this way, our study proposed a new form of CTC evaluation, unlike isolated analysis in previous studies, ${ }^{27,28,38,54,55}$ reinforcing the notion that the evaluation of CTC kinetics could be promising in the clinical follow-up of these patients. In addition, in the WT KRAS population, we may have a new tool to identify a population that is genuinely responsive to anti-EGFR therapy. Our results pave the way for the development of further studies that validate these data, which is essential to monitor the treatment of patients with $\mathrm{mCRC}$.

\section{Acknowledgments}

The authors thank São Paulo Research Foundation (FAPESP) for the financial support (grants: 2012/01273-8 and 2013/21730-7). They also thank Luciana M.M Ocea for the help with blood collection of some of the patients included in this study.

\section{Disclosure}

The authors report no conflicts of interest in this work.

\section{References}

1. Ferlay J, Soerjomataram I, Ervik M, et al. GLOBOCAN 2012 v1.0, Cancer Incidence and Mortality Worldwide: IARC CancerBase No. 11. Lyon, France: International Agency for Research on Cancer; 2013.

2. Lee WS, Yun SH, Chun HK, et al. Pulmonary resection for metastases from colorectal cancer: prognostic factors and survival. Int $J$ Colorectal Dis. 2007;22(6):699-704.

3. Van Cutsem E, Nordlinger B, Adam R, et al; European Colorectal Metastases Treatment Group. Towards a pan-European consensus on the treatment of patients with colorectal liver metastases. Eur J Cancer. 2006;42(14):2212-2221.

4. Yoo PS, Lopez-Soler RI, Longo WE, Cha CH. Liver resection for metastatic colorectal cancer in the age of neoadjuvant chemotherapy and bevacizumab. Clin Colorectal Cancer. 2006;6(3):202-207.

5. Chang GJ. Challenge of primary tumor management in patients with stage IV colorectal cancer. J Clin Oncol. 2012;30:3165-3166.

6. Heinemann V, von Weikersthal LF, Decker T, et al. FOLFIRI plus cetuximab versus FOLFIRI plus bevacizumab as first-line treatment for patients with metastatic colorectal cancer (FIRE-3): a randomised, open-label, phase 3 trial. Lancet Oncol. 2014;15(10):1065-1075.

7. Loupakis F, Cremolini C, Masi G, et al. Initial therapy with FOLFOXIRI and bevacizumab for metastatic colorectal cancer. N Engl J Med. 2014; 371(17):1609-1618.

8. Van Cutsem E, Rivera F, Berry S, et al; First BEAT Investigators. Safety and efficacy of first-line bevacizumab with FOLFOX, XELOX, FOLFIRI and fluoropyrimidines in metastatic colorectal cancer: the BEAT study. Ann Oncol. 2009;20(11):1842-1847.

9. Gustavsson B, Carlsson G, Machover D, et al. A review of the evolution of systemic chemotherapy in the management of colorectal cancer. Clin Colorectal Cancer. 2015;14(1):1-10.

10. Grothey A, Sugrue MM, Purdie DM, et al. Bevacizumab beyond first progression is associated with prolonged overall survival in metastatic colorectal cancer: results from a large observational cohort study (BRiTE). J Clin Oncol. 2008;26(33):5326-5334.

11. Karapetis CS, Khambata-Ford S, Jonker DJ, et al. K-ras mutations and benefit from cetuximab in advanced colorectal cancer. $N$ Engl J Med. 2008;359(17):1757-1765.

12. Chibaudel B, Tournigand C, André T, de Gramont A. Therapeutic strategy in unresectable metastatic colorectal cancer. Ther Adv Med Oncol. 2012;4(2):75-89.

13. Köhne CH, Cunningham D, Di Costanzo F, et al. Clinical determinants of survival in patients with 5-fluorouracil-based treatment for metastatic colorectal cancer: results of a multivariate analysis of 3825 patients. Ann Oncol. 2002;13(2):308-317.

14. Kamiyama H, Noda H, Konishi F, Rikiyama T. Molecular biomarkers for the detection of metastatic colorectal cancer cells. World J Gastroenterol. 2014;20(27):8928-8938.

15. Devriese LA, Voest EE, Beijnen JH, Schellens JH. Circulating tumor cells as pharmacodynamic biomarker in early clinical oncological trials. Cancer Treat Rev. 2011;37(8):579-589.

16. Yap TA, Lorente D, Omlin A, Olmos D, de Bono JS. Circulating tumor cells: a multifunctional biomarker. Clin Cancer Res. 2014;20(10): 2553-2568.

17. Gorges TM, Pantel K. Circulating tumor cells as therapy-related biomarkers in cancer patients. Cancer Immunol Immunother. 2013;62(5): 931-939.

18. Stebbing J, Jiao LR. Circulating tumor cells as more than prognostic markers. Lancet Oncol. 2009;10:1138-1139.

19. Cristofanilli M, Hayes DF, Budd GT, et al. Circulating tumor cells: a novel prognostic factor for newly diagnosed metastatic breast cancer. $J$ Clin Oncol. 2005;23(7):1420-1430.

20. de Bono JS, Scher HI, Montgomery RB, et al. Circulating tumor cells predict survival benefit from treatment in metastatic castration-resistant prostate cancer. Clin Cancer Res. 2008;14(19):6302-6309.

21. Cristofanilli M, Budd GT, Ellis MJ, et al. Circulating tumor cells, disease progression, and survival in metastatic breast cancer. $N$ Engl $J$ Med. 2004;351(8):781-791. 
22. Beije N, Jager A, Sleijfer S. Circulating tumor cell enumeration by the CellSearch system: the clinician's guide to breast cancer treatment? Cancer Treat Rev. 2015;41(2):144-150.

23. Goldkorn A, Ely B, Quinn DI, et al. Circulating tumor cell counts are prognostic of overall survival in SWOG S0421: a phase III trial of docetaxel with or without atrasentan for metastatic castration-resistant prostate cancer. J Clin Oncol. 2014;32(11):1136-1142.

24. Smerage JB, Barlow WE, Hortobagyi GN, et al. Circulating tumor cells and response to chemotherapy in metastatic breast cancer: SWOG S0500. J Clin Oncol. 2014;32(31):3483-3489.

25. de Albuquerque A, Kubisch I, Stolzel U, et al. Prognostic and predictive value of circulating tumor cell analysis in colorectal cancer patients. J Transl Med. 2012;10:222.

26. Otsuka K, Imai H, Soeda H, Komine K, Ishioka C, Shibata H. Practical utility of circulating tumor cells as biomarkers in cancer chemotherapy for advanced colorectal cancer. Anticancer Res. 2013;33: 625-629.

27. Tol J, Koopman M, Miller MC, et al. Circulating tumor cells early predict progression-free and overall survival in advanced colorectal cancer patients treated with chemotherapy and targeted agents. Ann Oncol. 2010;21:1006-1012.

28. Cohen SJ, Punt CJ, Iannotti N, et al. Relationship of circulating tumor cells to tumor response, progression-free survival, and overall survival in patients with metastatic colorectal cancer. J Clin Oncol. 2008;26(19): 3213-3221.

29. Lankiewicz S, Zimmermann S, Hollmann C, Hillemann T, Greten TF. Circulating tumor cells as a predictive factor for response to systemic chemotherapy in patients with advanced colorectal cancer. Mol Oncol. 2008;2:349-355.

30. Krebs MG, Hou JM, Sloane R, et al. Analysis of circulating tumor cells in patients with non-small cell lung cancer using epithelial markerdependent and -independent approaches. J Thorac Oncol. 2012;7(2): 306-315.

31. Krebs MG, Metcalf RL, Carter L, Brady G, Blackhall FH, Dive C. Molecular analysis of circulating tumour cells - biology and biomarkers. Nat Rev Clin Oncol. 2014;11:129-144.

32. Farace F, Massard C, Vimond N, et al. A direct comparison of CellSearch and ISET for circulating tumour-cell detection in patients with metastatic carcinomas. Br J Cancer. 2011;105(6):847-853.

33. Khoja L, Shenjere P, Hodgson C, et al. Prevalence and heterogeneity of circulating tumour cells in metastatic cutaneous melanoma. Melanoma Res. 2014;24(1):40-46.

34. Eisenhauer EA, Therasse P, Bogaerts J, et al. New response evaluation criteria in solid tumours: revised RECIST guideline (version 1.1). Eur J Cancer. 2009;45:228-247.

35. Chinen L, Mello C, Abdallah E, et al. Isolation, detection, and immunomorphological characterization of circulating tumor cells (CTCs) from patients with different types of sarcoma using isolation by size of tumor cells: a window on sarcoma-cell invasion. Onco Targets Ther. 2014;7: 1609-1617.

36. Van Cutsem E, Cervantes A, Nordlinger B, Arnold D. Metastatic colorectal cancer: ESMO clinical practice guidelines for diagnosis, treatment and follow-up. Ann Oncol. 2014;25:1-9.

37. Romiti A, Raffa S, Di Rocco R, et al. Circulating tumor cells count predicts survival in colorectal cancer patients. J Gastrointestin Liver Dis. 2014;23(3):279-284.

38. Almufti R, Wilbaux M, Oza A, et al. A critical review of the analytical approaches for circulating tumor biomarker kinetics during treatment. Ann Oncol. 2015;25(1):41-56.
39. Wallwiener M, Riethdorf S, Hartkopf AD, et al. Serial enumeration of circulating tumor cells predicts treatment response and prognosis in metastatic breast cancer: a prospective study in 393 patients. BMC Cancer. 2014;14:512.

40. Cohen SJ, Punt CJ, Iannotti N, et al. Prognostic significance of circulating tumor cells in patients with metastatic colorectal cancer. Ann Oncol. 2009;20(7):1223-1229.

41. Huang X, Gao P, Song Y, et al. Relationship between circulating tumor cells and tumor response in colorectal cancer patients treated with chemotherapy: a meta-analysis. BMC Cancer. 2014;14:976.

42. Vona $\mathrm{G}$, Sabile A, Louha M, et al. Isolation by size of epithelial tumor cells. A new method for the immunomorphological and molecular characterization of circulating tumor cells. Am J Pathol. 2000;156(1): 57-63.

43. Hofman V, Long E, Ilie M, et al. Morphological analysis of circulating tumor cells in patients undergoing surgery for non-small cell lung carcinoma using the isolation by size of epithelial tumor cell (ISET) method. Cytopathol. 2012;23:30-38.

44. Paterlini-Bréchot P. Circulating tumor cells: who is the killer? Cancer Microenviron. 2014;7(3):161-176.

45. Aggarwal C, Meropol NJ, Punt CJ, et al. Relationship among circulating tumor cells, CEA and overall survival in patients with metastatic colorectal cancer. Ann Oncol. 2013;24(2):420-428.

46. Kuboki Y, Matsusaka S, Minowa S, et al. Circulating tumor cell (CTC) count and epithelial growth factor receptor expression on CTCs as biomarkers for cetuximab efficacy in advanced colorectal cancer. Anticancer Res. 2013;33(9):3905-3910.

47. Sastre J, Vidaurreta M, Gómez A, et al; Spanish Cooperative Group for the Treatment of Digestive Tumors. Prognostic value of the combination of circulating tumor cells plus KRAS in patients with metastatic colorectal cancer treated with chemotherapy plus bevacizumab. Clin Colorectal Cancer. 2013;12(4):280-286.

48. Douillard JY, Oliner KS, Siena S, et al. Panitumumab-FOLFOX4 treatment and RAS mutations in colorectal cancer. N Engl J Med. 2013; 369(11):1023-1034.

49. Malka D, Boige V, Jacques $\mathrm{N}$, et al. Clinical value of circulating endothelial cell levels in metastatic colorectal cancer patients treated with first-line chemotherapy and bevacizumab. Ann Oncol. 2012;23(4):919-927.

50. Bidard FC, Mathiot C, Degeorges A, et al. Clinical value of circulating endothelial cells and circulating tumor cells in metastatic breast cancer patients treated first line with bevacizumab and chemotherapy. Ann Oncol. 2010;21(9):1765-1771.

51. Manzoni M, Comolli G, Torchio M, Mazzini G, Danova M. Circulating endothelial cells and their subpopulations: role as predictive biomarkers in antiangiogenic therapy for colorectal cancer. Clin Colorectal Cancer. 2015;14(1):11-17.

52. Nicolazzo C, Massimi I, Lotti LV, et al. Impact of chronic exposure to bevacizumab on EpCAM-based detection of circulating tumor cells. Chin J Cancer Res. 2015;27(5):491-496.

53. Budd GT, Cristofanilli M, Ellis MJ, et al. Circulating tumor cells versus imaging-predicting overall survival in metastatic breast cancer. Clin Cancer Res. 2006;12:6403-6409.

54. Raimondi C, Gradilone A, Naso G, Cortesi E, Gazzaniga P. Clinical utility of circulating tumor cell counting through CellSearch ${ }^{\mathbb{}}$ : the dilemma of a concept suspended in Limbo. Onco Targets Ther. 2014;17:619-625.

55. Gazzaniga P, Raimondi C, Gradilone A, et al. Circulating tumor cells in metastatic colorectal cancer: do we need an alternative cutoff? J Cancer Res Clin Oncol. 2013;139(8):1411-1416. 
OncoTargets and Therapy

\section{Publish your work in this journal}

OncoTargets and Therapy is an international, peer-reviewed, open access journal focusing on the pathological basis of all cancers, potential targets for therapy and treatment protocols employed to improve the management of cancer patients. The journal also focuses on the impact of management programs and new therapeutic agents and protocols on

patient perspectives such as quality of life, adherence and satisfaction. The manuscript management system is completely online and includes a very quick and fair peer-review system, which is all easy to use. Visit http://www.dovepress.com/testimonials.php to read real quotes from published authors.

Submit your manuscript here: http://www.dovepress.com/oncotargets-and-therapy-journal 